\title{
MUERTE SÚBITA POR RÚPTURA ESPLÉNICA EN UN TERNERO. DESCRIPCIÓN PATOLÓGICA
}

\section{SUDDEN DEATH IN A CALF BY SPLENIC RUPTURE PATHOLOGY DESCRIPTION}

PERDOMO, A. SANDRA ${ }^{1}$ MVZ., CARDONA, A. JOSE ${ }^{2,4 *}$ MSc., MONTES, V. DONICER ${ }^{3}$ MSC.

${ }^{1}$ Universidad de Córdoba, Departamento de Ciencias Pecuarias, Semillero de Estudios e Investigaciones en Medicina de Grande Animales, Grupo MEGA, Histotécnica Laboratorio de Patología, Estudiante de Maestría en Ciencias Veterinarias del Trópico, Montería, Colombia. ${ }^{2}$ Universidad de Córdoba, Departamento de Ciencias Pecuarias, Semillero de Estudios e Investigaciones en Medicina de Grandes Animales, Grupo MEGA, Profesor de Medicina y Clínica de Grandes Animales, Montería, Colombia. Estudiante de Doctorado en Medicina

Veterinaria, Universidad Federal de Viçosa, Brasil. ${ }^{3}$ Universidad de Sucre,

Facultad de Ciencias Agropecuarias, Departamento de Zootecnia. Sincelejo, Colombia. Grupo de Investigación en Mejoramiento y Reproducción Animal, Estudiante de Doctorado en Genética y Mejoramiento Animal, Universidad Estadual Paulista (Unesp)SP, Brasil. ${ }^{4}$ Bolsista do Programa Estudantes-Convênio de Pós-Graduação - PEC-PG, da CAPES/CNPq - Brasil.

*Correspondencia: cardonalvarez@hotmail.com

Recibido: 08-16-2013; aceptado: 22-10-2013.

\section{Resumen}

El objetivo de este estudio es reportar un caso de muerte súbita en un ternero de 7 días por ruptura esplénica en el departamento de Córdoba (Colombia). Fueron analizados: la historia clínica, los hallazgos de necropsia y el estudio histopatológico con tinción de Hematoxilina-Eosina. El animal fue dejado en la tarde del día anterior en condiciones de aparente normalidad y al día siguiente en horas de la mañana el operario reportó decaimiento y muerte unos minutos después. En la necropsia, al examen del cadáver se determinó mucosas pálidas y marcado signo de deshidratación, debido a la depresión ocular y a la resequedad de los tejidos, en la cavidad abdominal se encontró marcada colección de líquido sanguinolento libre acompañado de aumento focalizado del bazo debido a un hematoma subcapsular, posiblemente de origen traumático con ruptura del borde mayor, causando un shock hipovolémico de tipo hemorrágico.

Palabras clave: Bazo, hemorragia, hemoperitoneo.

\section{Abstract}

The objective of this study is to report a case of sudden death in a calf 7-day splenic rupture in the department of Cordoba (Colombia). Were analyzed: the history, necropsy findings and histopathological examination with hematoxylineosin staining. The animal was left in the previous afternoon in apparent normality 
conditions and the following day in the morning the operator reported decay and death within minutes. At necropsy, the examination of the body was determined and marked pale mucous sign of dehydration, due to depression and dryness ocular tissues in the abdominal cavity was found accompanied by marked increase targeted hemoperitoneum due to a subcapsular hematoma, possibly with traumatic rupture of the largest edge, causing hemorrhagic hypovolemic shock type.

Key words: Spleen, bleeding, haemoperitoneum.

\section{Introducción}

La muerte súbita de los terneros puede ser el resultado de enfermedadades, como también de accidentes o de una acción intencionada por personas, y tiene un efecto pronunciado sobre la productividad en los animales, lo cual no solo compromete el bienestar animal sino también la economía, generando dudas acerca del correcto manejo en la producción (WAYNE et al., 1997). RADOSTITS et al., (2002) dice que la tasa de mortalidad de bovinos de hasta un mes de edad puede variar de 3 a $30 \%$ de acuerdo con las condiciones del rebaño y la propiedad en cuestión. BENAVIDES (2004), informa como principales causas de muerte súbita en los terneros entidades como la rabia bovina, botulismo, carbón bacteridiano (ántrax o carbunco), carbón sintomático, plantas tóxicas, fiebre de garrapatas, intoxicación por nitratos y nitritos o glucósidos cianogenéticos, deficiencia de tiamina, deficiencia de minerales, lesiones neurológicas asociadas a virus (particularmente herpesvirus) y mordeduras de serpientes, también WATANABE et al., (2010) indica que el fallecimiento espontaneo de estos animales también esta relacionado a la electrocución ya sea por las descargas eléctricas atmosférica (rayos), instalaciones inadecuadas de vallas eléctricas.

BRANCO (2008), reporto que los órganos afectados por traumas como el bazo también contribuyen como una causa de muerte súbita ya que el animal puede o no mostrar sinologías, y cuando no es tratada a tiempo se puede presentar hemoperitonitis por ruptura esplénica ocasionando shock hipovolémico de tipo hemorrágico, denominándose a este proceso como de tipo espontaneo, así mismo se encuentran las de tipo patológico, las cuales son predisponentes pero silenciosas, como sucede en casos de disturbios esplénicos que son identificados generalmente por medio de una alteración en el tamaño, forma y función del bazo constituyendo un proceso subyacente como neoplasias primarias o metastásicas, hiperplasia nodular, hematoma, abscesos, enfermedades inflamatorias e infecciosas (BIRCHARD y SHERDING, 2003). 
El bazo en el bovino tiene forma elíptica alongada, con ambas extremidades delgadas, redondeadas y del mismo tamaño. En el adulto el peso promedio, es de aproximadamente $900 \mathrm{~g}$ que representa el $0,16 \%$ del peso corporal. La longitud media es de $50 \mathrm{~cm}$., el ancho $15 \mathrm{~cm}$. y el grosor de unos 2 a $3 \mathrm{~cm}$ (VENZKE, 1982).

El bazo es el órgano linfático más grande del organismo, participa en la respuesta inmune primaria contra microorganismos y proteínas extrañas, posee una pulpa roja que ocupa más de la mitad del volumen del bazo, el cual se destaca por ser un filtro de sangre ya que es el sitio donde se destruyen los gerocitos y se extraen las inclusiones celulares de los reticulocitos y la pulpa blanca contiene macrófagos y linfocitos $B$ y $T$ que participan en el reconocimiento de microorganismos y proteínas extrañas como parte de la respuesta inmune primaria (el bazo es el principal productor de lgM, principalmente en la etapa neonatal) (VARGAS et al., 2013).

\section{Presentación del caso}

Fue reportada la muerte de un ternero en las instalaciones de la granja Berástegui de la Facultad de Medicina Veterinaria y Zootecnia de la Universidad de Córdoba, el cadáver del mismo fue trasladado a la sala de necropsia del área de patología animal. El caso correspondió a un ternero de 7 días de nacido, macho, mestizo y con un peso aproximado de $50 \mathrm{Kg}$. Se informó que el animal fue dejado en la tarde del día anterior en condiciones de aparente normalidad y al día siguiente en horas de la mañana el operario expresó que el animal presento decaimiento y muerte unos minutos después. Al examen macroscópico se evidencio palidez de las membranas mucosas, depresión ocular, resequedad del tejido subcutáneo, siendo estos hallazgos consistentes con anemia y deshidratación. Al examen del abdomen se encontró abundante líquido sanguinolento libre, compatible con hemoperitoneo (Fig. 1), acompañado de aumento focalizado del bazo con ruptura a nivel de su borde mayor (Fig. 2).

Fue tomada una muestra de $1 \mathrm{~cm}$ de espesor, de la periferia del hematoma del tejido esplénico, siendo fijada en formalina tamponada al 10\% durante 24 horas y después conservada en alcohol al 70\%, luego procesada con los métodos histológicos de rutina, cortadas en micrótomo a $5 \mu \mathrm{m}$ de espesor y teñidas con la coloración de Hematoxilina-Eosina en el laboratorio de patología de la Facultad de Medicina Veterinaria y Zootecnia de la Universidad de Córdoba, Colombia (Fig. 3). 


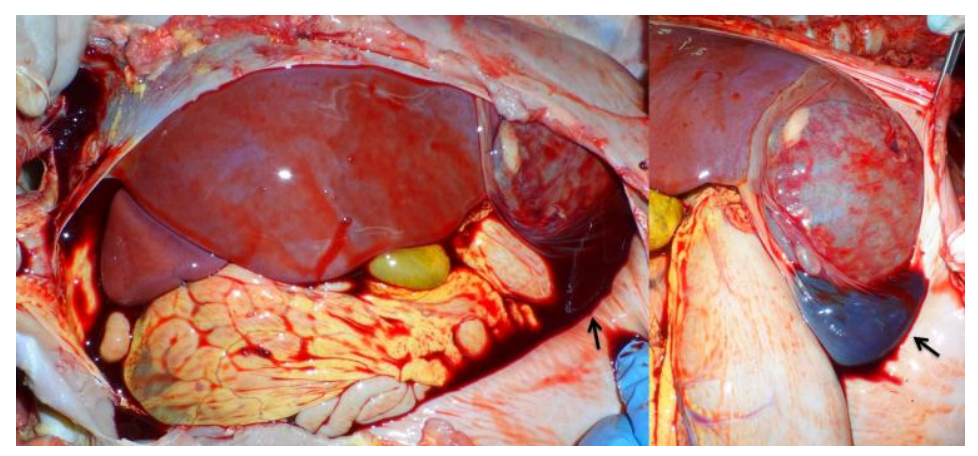

Figura 1. Obsérvese la presencia de sangre dispersa en la cavidad abdominal (ver flechas). Marcada cantidad de líquido sanguinolento libre compatible con Hemoperitoneo.

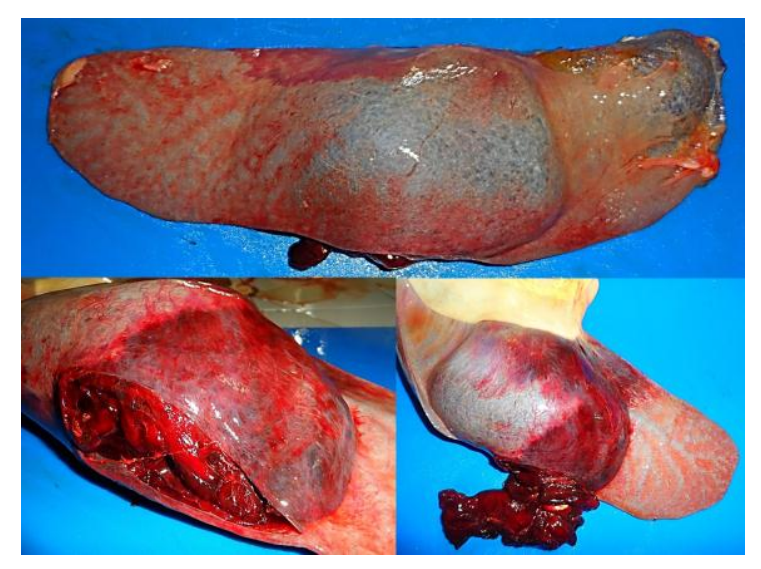

Figura 2. Nótese la ruptura del bazo a nivel del borde mayor, de aproximadamente $8 \mathrm{~cm}$ de largo con presencia de hematoma sub-capsular.

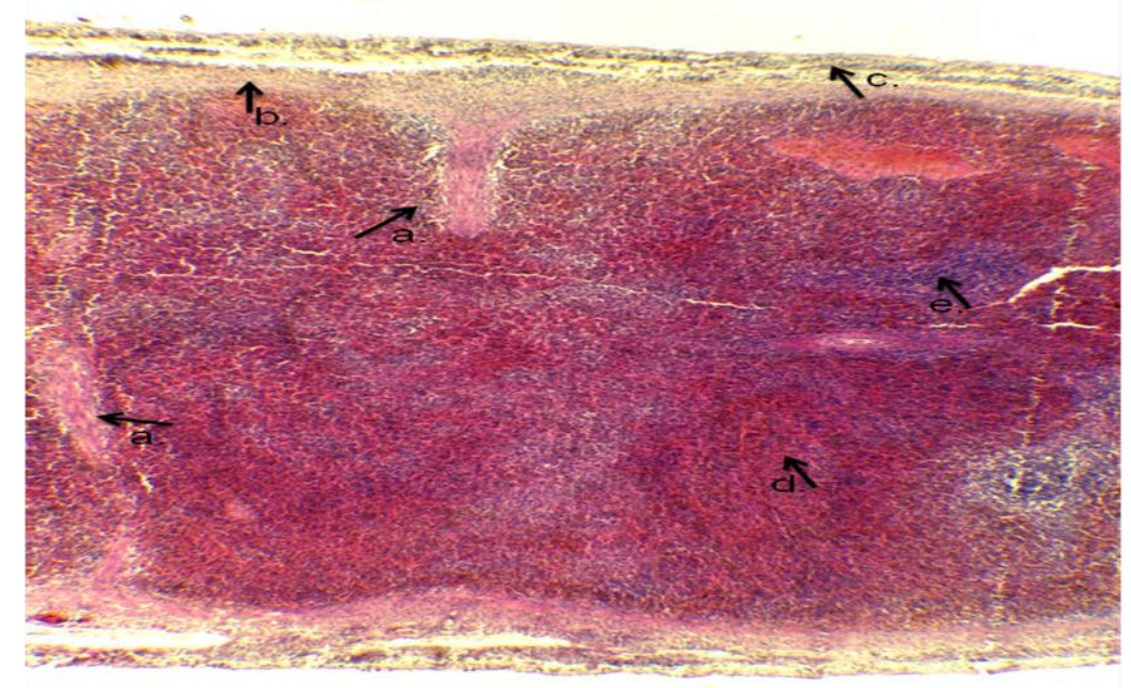

Figura 3. Corte histológico del bazo, observe la aparente normalidad del tejido, a) Trabécula b) Capsula c) Mesotelio d) Pulpa roja e) Pulpa blanca. Vista 10x. 


\section{Resultado y Discusión}

La muerte súbita se define como la condición donde los animales aparecen muertos de forma repentina o bien poseen una enfermedad de muy corto curso o sobreaguda; situación bajo la cual no se da tiempo para el desarrollo de lesiones patológicas, dificultando el diagnóstico, el cual debe basarse en la recolección de evidencia epidemiológica. En ocasiones hay animales que pueden presentar alteraciones con sintomatología de corta duración que no es observada por los vaqueros o el personal a cargo del manejo del ganado y por ende no es alcanzado a establecer el diagnóstico o instaurar algún tipo de tratamiento (BENAVIDES, 2004).

En la necropsia se encontró grandes cantidades de líquido sanguinolento en la cavidad abdominal del ternero, también se observó hematoma en la capsula del bazo, al extraer el órgano, en la cara parietal del bazo se observó ruptura de aproximadamente 8 centímetros en el borde mayor del órgano que según la escala de lesión esplénica en humanos descrita por MOORE et al., (1989) se clasificó en nivel III ya que presentó hematoma sub-capsular roto con hemorragia activa (Tabla 1).

Tabla 1. Escala de Lesión Esplénica (MOORE et al., 1989).

\begin{tabular}{|c|c|c|}
\hline & Grados & Descripción \\
\hline \multirow[b]{2}{*}{ I } & Hematoma & Subcapsular, no expansivo menor $10 \%$ superficie \\
\hline & Laceración & Desgarro capsular, no sangrante, menor $1 \mathrm{~cm}$ de profundidad. \\
\hline \multirow[t]{2}{*}{ II } & Hematoma & $\begin{array}{l}\text { Subcapsular, no expansivo, } 10-50 \% \text { superficie intra- } \\
\text { parenquimatoso, no expansivo, menor } 2 \mathrm{~cm} \text {. }\end{array}$ \\
\hline & Laceración & $\begin{array}{l}\text { Desgarro capsular, hemorragia activa, de } 1 \text { a } 3 \mathrm{~cm} \text { de } \\
\text { profundidad que no afecta vasos trabeculares. }\end{array}$ \\
\hline \multirow[t]{2}{*}{ III } & Hematoma & $\begin{array}{l}\text { Subcapsular, mayor } 50 \% \text { superficie o expansivo hematoma } \\
\text { capsular roto con hemorragia activa hematoma intra- } \\
\text { parenquimatoso mayor } 2 \mathrm{~cm} \text { o expansivo. }\end{array}$ \\
\hline & Laceración & $\begin{array}{l}\text { De más de } 3 \mathrm{~cm} \text { de profundidad en el parénquima o afecta } \\
\text { vasos trabeculares. }\end{array}$ \\
\hline & Hematoma & Hematoma intra-parenquimaoso roto con hemorragia activa. \\
\hline IV & Laceración & $\begin{array}{l}\text { Laceración que afecta vasos segmentados o hiliares, } \\
\text { produciendo desvascularización mayor (más } 25 \% \text { del bazo). }\end{array}$ \\
\hline
\end{tabular}




\begin{tabular}{lll}
\hline V & $\begin{array}{l}\text { hematoma } \\
\text { laceración }\end{array}$ & $\begin{array}{l}\text { Estallido esplénico. } \\
\text { Lesión vascular hiliar que desvasculariza el bazo. }\end{array}$ \\
\hline
\end{tabular}

La ruptura esplénica se presenta en forma espontanea o con mínimo trauma en casos de esplenomegalia, pudiendo ser una complicación catastrófica de aparición súbita que conduce a hemorragia masiva intra- abdominal, choque y muerte en pocos minutos a menos que se proceda de manera inmediata a la reanimación e intervención quirúrgica (VARGAS et al., 2013).

ESPINOZA et al. (1995), informa que la ruptura esplénica indudablemente se expresa con hemoperitoneo, la mayor parte de las veces (excepto en casos de hematomas sub-capsulares o intra-parenquimatosos localizados) y será la magnitud de este lo que determine el espectro clínico de presentación: desde un paciente asintomático o mínimos signos de complicación intra-abdominal hasta un shock profundo por estallido del bazo.

RENZULLI et al. (2009), identificaron seis grupos etiológicos de la ruptura espontanea del bazo, entre las cuales se encuentran las neoplásicas, infecciosas, inflamatorias, no infecciosa, farmacológicas relacionada con tratamientos y trastornos mecánicos, mientras que para GORG et al. (2003), los casos de ruptura esplénica incluyen los trastornos de la coagulación, trombocitopenia, hipertensión portal, vasculitis, trombosis venosa del bazo y lesiones esplénicas focales.

En la evaluación histopatológica de las muestras se observó aparente normalidad histológica, por lo que se descarta el posible origen inflamatorio, ya que no hubo tiempo para que el organismo respondiera ante este proceso, pudiendo orientar a que la posible causa de la ruptura del bazo fue de origen traumático y agudo, concordando con lo informado por BENAVIDES (2004).

Basados en los datos anamnesicos, el examen post morten del cadáver, la caracterización macroscópica de las lesiones encontradas en la necropsia y la aparente normalidad histológica se puede concluir, que el aumento focalizado correspondió a un hematoma subcapsular, posiblemente de origen traumático con ruptura del borde mayor conllevando a hemorragia abdominal y muerte por shock hipovolémico de tipo hemorrágico, ya que en casos de hemorragia severa, el organismo entra inicialmente en un estado de emergencia hemodinámica, tratando de ser controlado por los mecanismos compensatorios como la reacción cardiovascular y neuroendocrina, sin embargo, en los casos agudos no se alcanza a controlar por estos mecanismos y si la pérdida de sangre supera el $20 \%$, el organismo entra en un estado de shock hipovolémico por pérdida aguda de 
sangre, siendo la disminución del gasto cardiaco la causa de la muerte, por la incapacidad del sistema cardiovascular para perfundir en los tejidos bajo los límites normales de presión y flujo (GARCíA, 2009).

\section{Agradecimientos}

Al área de patología de la Facultad de Medicina Veterinaria y Zootecnia de la Universidad de Córdoba, por su apoyo incondicional en el desarrollo del caso y en el procesamiento y análisis de las muestras.

Conflictos de intereses: El manuscrito fue preparado y revisado con la participación de todos los autores, quienes declaramos que no existe ningún conflicto de intereses que ponga en riesgo la validez de los resultados presentados.

\section{Referencias}

BRANCO, M. 2008. Hematoma de baço- Relato de Caso. Monografia de conclusão do curso de Especialização Latu sensu. Universidad Castelo Branco, Rio de Janeiro.

BENAVIDES, E. 2004. Causas de muerte súbita en bovinos en pastoreo en las sabanas de América Tropical. Rev Col Cienc Pec. 17(2):182-192.

BIRCHARD, S.J.; SHERDING, R. G., 2003. Manual Saunders: Clínica de pequenos animais. 2. ed. São Paulo: Roca.

DISTILIO, G. 2005. Ruptura esplénica espontanea secundaria a amiloidosis, Rev. Medicina 65 (2): 538-542.

ESPINOZA, R.; AGUILERA, H.; PLAZA DE LOS REYES M., et al. 1995. Cirugía del Traumatismo Esplénico Rev. Chil. Cir 47:75-80.

GARCÍA, P. 2009. Shock hemorrágico. Rev. R. Acad. Cienc. Exact. Fís. Nat. 103 (1):1-39.

GORG, C.; COLLE, J.; GORG, K.; PRINZ, H.; ZUGMAIER G. 2003. "Spontaneous rupture of the spleen: ultrasound patterns, diagnosis and follow-up," British Journal of Radiology 76 (91):704- 711.

MEBIUS, R.; KRAAL, G. 2005. Structure and function of the spleen; nature publishing group Vol 5. URL disponible en: www.nature.com/reviews/immunol 
MOORE, E.;SHACKFORD, S.; PACHTER L. 1989. Organ Injury Scaling: Spleen, Liver and Kidnay. J. Trauma 25: 1664-1666.

RADOSTITS, O. M.; GAY, C. C.; BLOOD, D. 2002. Clinica veterinária. 9 ed. Rio de Janeiro: Guanabara Koogan.

RENZULLI, P.; HOSTETTLER, A.; SCHOEPFER, M.; GLOOR, B.; CANDINAS, D. 2009. "Systematic review of atraumatic splenic rupture," British Journal of Surgery 96 (10):1114-1121.

VARGAS, P.;HURTADO, R.; VILLALOBOS, J. 2013. Esplenomegalia. Revista de la Facultad de Medicina de la UNAM 56 (2):37- 45.

VENZKE, W. G. 1982. Timo. En: GETTY, R. SISSON y GROSSMAN. Anatomía de los Animales Domésticos. Quinta edición, Salvat. Barcelona.

WATANABE, T. et al., 2010, Fulguração como causa de morte em bovinos no Estado do Rio Grande do Sul1 Pesq. Vet. Bras. 30(3):243-245

WAYNE, S.; MEEK A. H.; WILLEBERG, P. 1997. Epidemiología veterinaria. Principios y métodos. Editorial Acribia S.A., Zaragoza, España. 